\title{
Divertículo de Meckel. Reporte de casos
}

\author{
Meckel's diverticulum. Case reports
}

\section{Agueda Arriola López, * Norma González**}

\section{RESUMEN}

El divertículo de Meckel es el defecto congénito más frecuente del tracto gastrointestinal. Se trata de un remanente del conducto onfalomesentérico, una estructura que conecta el saco vitelino primitivo al intestino medio durante el desarrollo del feto. El conducto onfalomesentérico se oblitera entre la quinta y séptima semanas de gestación. Generalmente se encuentra localizado en el último metro de intestino delgado en el borde antimesentérico.

Puede ser asintomático o manifestarse como un cuadro de abdomen agudo. Las complicaciones en un escaso número de pacientes pueden ser: infección, sangrado digestivo, obstrucción y raramente transformación maligna.

A continuación, se presentan 4 casos de pacientes pediátricos con diagnóstico postoperatorio de divertículo de Meckel del Instituto Hondureño de Seguridad Social de San Pedro Sula, durante el período 2012-2014. El presente artículo se realizó con la finalidad de brindar una lección clínica a los colegas y permitir ampliar sus conocimientos sobre esta entidad.

\section{PALABRAS CLAVE}

Divertículo lleal.

\section{ABSTRACT}

Meckel's diverticulum is the most common birth defect of the gastrointestinal tract, it is a remnant of the omphalomesenteric duct, a structure that connects the yolk sac to the primitive midgut during fetal development. Omphalomesenteric obliterates the canal between the fifth

\footnotetext{
*Residente de Segundo año Postgrado de Pediatría Universidad Nacional Autónoma de Honduras Valle de Sula

** Pediatra Gastroenteróloga. Pediatra de Guardia Hospital Regional del Norte Instituto Hondureño de Seguridad Social

Dirigir correspondencia a: aaarriola_lopez@hotmail.com

Recibido: 17 de agosto 2016 Aprobado: 19 de noviembre 2016
}

and seventh weeks of gestation. It is generally located in the last meter of small intestine in its antimesial side. It may be asymptomatic or manifest as an acute appendicitis and can be complicated by bleeding, diverticulitis, obstruction and rarely with malignant transformation.

Four children diagnosed with Meckel's diverticulum at the Honduran Social Security Institute are presented, to expand knowledge about this entity.

\section{KEY WORDS}

Meckel diverticulum.

\section{INTRODUCCION}

El divertículo de Meckel fue descrito originalmente por el padre de la cirugía alemana, Guilhelmus Fabricius Hildanus (1560-1634) en 1598. En 1742, otro divertículo estrangulado en una hernia inguinal fue reportado por Littré.(1) Sin embargo, fue hasta 1809 que el anatomista alemán Johann Friedrich Meckel (1781-1833). describió las bases embriológicas del divertículo, demostrando que es resultado de la atrofia incompleta del conducto onfalomesentérico o vitelino. Fue casi 100 años después, que Salzer y Deetz permitieron comprender la complicación hemorrágica del divertículo de Meckel con el descubrimiento de la mucosa ectópica gástrica y la ulceración asociada. ${ }^{(2)}$ En 1898, Kuttner reportó la intususcepción de intestino delgado secundario a la invaginación de un divertículo de Meckel.(3)

Su prevalencia oscila en 1 a 2\% de la población en general.(1) El divertículo de Meckel es sintomático en $4-6 \%$ de los pacientes. La proporción de hombres y mujeres en casos sintomáticos se informa que es 3:1.(4) El diagnóstico suele hacerse en la infancia, entre el $50 \%$ y $60 \%$ de los pacientes que desarrollan síntomas son menores de 2 años de edad.(2) 
Su riego sanguíneo proviene la mayoría de las veces de un remanente de la arteria vitelina derecha procedente a su vez de la arteria mesentérica superior; normalmente se localiza en los últimos 100 centímetros proximales a la válvula ileocecal, aunque ha sido descrito tan lejano como en el ligamento de Treitz; el tamaño promedio se describe como de tres centímetros. $^{(3)}$

Es bien conocido el hecho de que en la mitad de los casos aproximadamente tiene mucosa heterotópica gástrica, además en un $16 \%$ de los casos se ha descrito tejido acinar pancreático y muy raramente glándulas de Brunner, mucosa colónica, tejido hepatobiliar o combinaciones de varios tejidos. ${ }^{(4-8)}$

Los tumores malignos que surgen del divertículo de Meckel son raros y hay pocos casos informados de tumores carcinoides, tumor del estroma gastrointestinal (GIST), carcinoma de células en anillo de sello y el adenocarcinoma. Linfomas que se desarrollan en el divertículo de Meckel son extremadamente raros y hay pocos casos descritos en la literatura incluidos linfoma de Burkitt, linfoma linfoblástico plasmocitoide, un linfoma de células $B$ sin mayor clasificación y un linfoma $B$ de zona marginal extranodal. ${ }^{(9,10)}$

El método diagnóstico más preciso en la detección del divertículo de Meckel es la gammagrafía con tecnecio 99 de mucosa gástrica ectópica, ya que dicho radiofármaco tiene una alta afinidad por la mucosa gástrica $y$, tras su aplicación intravenosa, se realiza una centellografía para identificar la localización de mucosa ectópica. Uno de los beneficios de la gammagrafía es que es un estudio no invasivo que en niños tiene una sensibilidad del $85-97 \%$ y una especificidad del $95 \%$. La precisión de dicho estudio se eleva al administrar al paciente un antagonista del receptor de histamina II, vía intravenosa entre 24 a 48 horas antes del estudio. ${ }^{(11)}$
El tratamiento se basa en la resección del divertículo de Meckel, por laparotomía o laparoscopia, ${ }^{(12)}$ sintomático o no, dicha cirugía correctiva puede realizarse mediante diverticulectomía o resección segmentaria con anastomosis; ${ }^{(13)}$ ambos cuentan con un pronóstico a corto y largo plazo excelentes. ${ }^{(11,14,15)}$

\section{CASO 1}

Masculino de 5 meses de edad, con historia referida por la madre de distensión abdominal de 3 días de evolución, fiebre de 1 día de evolución, múltiples episodios de vómitos de contenido alimentario y bilioso y una deposición sanguinolenta; al examen físico se palpó masa de $7 \times 7 \mathrm{~cm}$ en mesogástrico no dolorosa. Se realizó laparotomía exploratoria en la que se encontró invaginación intestinal ileo-colónica sin compromiso vascular y divertículo de Meckel a $30 \mathrm{~cm}$ de la válvula ileocecal por lo que se le realiza reducción manual de invaginación intestinal, resección de divertículo de Meckel y apendicetomía profiláctica.

\section{CASO 2}

Masculino de 2 años, con historia de vómitos postprandiales de 4 horas de evolución, 5 episodios de contenido alimentario y uno de contenido bilioso, dolor abdominal del mismo tiempo de evolución, cuadro acompañado con signos de deshidratación. Se practicó laparotomía exploratoria por cuadro de obstrucción intestinal que reveló un divertículo de Meckel de $5 \mathrm{~cm}$ de longitud, no perforado, a $50 \mathrm{~cm}$ de válvula ileocecal más banda que ocasiona vólvulo de intestino medio; se libera banda, con lo que se logra resolución de vólvulo, resección en cuña de Meckel y apendicetomía profiláctica.

\section{CASO 3}

Masculino de 8 años de edad, con cuadro de dolor abdominal de 3 días de evolución, localizado en cuadrante inferior derecho, tipo cólico sin irradiación acompañado de distensión abdominal, paro de evacuaciones de 1 día de evolución, además de vómitos de contenido 
alimentario y biliar; se practicó laparotomía exploratoria en la que se encuentra alrededor de $200 \mathrm{ml}$ de líquido libre en cavidad abdominal, divertículo de Meckel perforado a nivel de tercio distal, bridas y adherencias a nivel de íleon terminal, por lo que se procede a realizar diverticulectomía y resección intestinal termino-terminal $5 \mathrm{~cm}$ a cada lado, apendicetomía profiláctica y lavado quirúrgico. Paciente es reintervenido 48 horas después por presentar síndrome compartamental abdominal en fase inicial; 7 días más tarde se le realiza drenaje de seroma paraumbilical derecho. Reingresa a los 14 días, y permanece hospitalizado 72 horas, por cuadro de suboclusión intestinal por bridas el cual resolvió con manejo médico.

\section{CASO 4}

Femenina de 9 años con sintomatología de dolor abdominal de 2 días de evolución en epigastrio irradiado a hipogastrio y fiebre del mismo tiempo de evolución, no vómitos. Se realiza laparotomía encontrando apéndice con hallazgos de periapendicitis, liquido turbio en hueco pélvico, plastrón de epiplón sobre divertículo de Meckel de aproximadamente $1.5 \mathrm{~cm}$ de longitud perforado en el vértice; se le practica diverticulectomía, apendicetomía y lavado quirúrgico. Reintervenida 7 meses después por cuadro de obstrucción intestinal por bridas; por lo que es sometida a laparotomía, bridectomía y descompresión intestinal.

\section{DISCUSION}

Comúnmente se hace referencia al divertículo de Meckel con la «regla de los dos» para describir su epidemiología: ${ }^{(2,4,11)}$

- Ocurre en el 2\% de la población.

- Relación hombre/mujer 2:1.

- Localización frecuente, a dos pies $(60 \mathrm{~cm})$ de la válvula ileocecal en el borde antimesentérico.

- Usualmente mide dos cm de diámetro.

- Generalmente mide dos pulgadas (cinco cm) de longitud.

- Involucra dos tipos de tejido ectópico (gástri- co y pancreático).

- Aproximadamente el $2 \%$ de los individuos con un divertículo de Meckel desarrollan una complicación a lo largo de su vida.

- Se presenta habitualmente antes de los dos años de edad.

Tres de los cuatro casos correspondieron a pacientes del género masculino, concordando con lo referido en la literatura, en cuanto a su frecuencia según genero. ${ }^{(4,11,16)}$

El 33\% de los pacientes presentan sintomatología, mientras que un $16 \%$ de los pacientes permanecen asintomáticos, siendo el divertículo de Meckel un hallazgo incidental durante un procedimiento quirúrgico indicado por una patología distinta. ${ }^{(2)}$

Cuando el divertículo de Meckel presenta manifestaciones clínicas, éstas suelen ser inespecíficas y por tanto se dificulta el diagnóstico. La presencia de síntomas generalmente obedece al desarrollo de complicaciones. ${ }^{(17)} \mathrm{El}$ riesgo de desarrollar complicaciones es de 4.2\%. La incidencia de complicaciones disminuye conforme aumenta la edad de los pacientes. ${ }^{(18)}$ La complicación más frecuente en niños es la hemorragia digestiva baja, usualmente se presenta como hematoquezia. (2) Otras complicaciones incluyen ulceración, obstrucción intestinal, perforación, intususcepción, fístula vesicodiverticular, y la malignidad. ${ }^{(19)}$ Coincidiendo con los casos reportados, ya que dos de ellos cursaron con perforación, uno con intususcepción, además de un cuarto caso en el que el divertículo de Meckel fue un hallazgo incidental.

En algunos pacientes que presentan diverticulitis (causante del $20 \%$ de las complicaciones), los síntomas que llevan al diagnóstico del divertículo de Meckel son similares a los síntomas de apendicitis, pueden incluir fiebre, dolor, náusea y vómito, como lo encontrado en nuestros casos. A la exploración física se encuentra sensibilidad a la palpación, rigidez y signo de rebote. (2) Uno de nuestros pacientes presentó 
cuadro clínico de apendicitis y plastrón de epiplón sobre el divertículo, y 3 de ellos presentaron signos clínicos de obstrucción intestinal.

El diagnóstico debe ser considerado en cualquier paciente con malestar abdominal, náusea y vómito o hemorragia intestinal. ${ }^{(2,20)} \mathrm{El}$ cuadro clínico que acompaña al divertículo de Meckel puede ser indistinguible del que se presenta en otras patologías gastrointestinales. El diagnóstico diferencial más común es apendicitis; no obstante, la sintomatología puede confundirse con otras enfermedades comunes como úlcera péptica, gastroenteritis, cólico biliar, diverticulitis e incluso intolerancia a la lactosa. ${ }^{(21)}$ ( Ver cuadro No. 1).

El método diagnóstico más preciso en la detección del divertículo de Meckel es el estudio con tecnecio-99m pertecnetato. Sin embargo, depende de la captación por la mucosa gástrica heterotópica, siendo útil entonces para el diagnóstico en pacientes sintomáticos, por ejemplo, con hemorragia intestinal. ${ }^{(2)}$

En conclusión, el Divertículo de Meckel es la anomalía congénita más común del tracto gastrointestinal. Clinicamente silente es un hallazgo incidental en la exploración quirúrgica por otra patología. Cuando esta sintomático se presenta como dolor abdominal o sangrado digestivo u obstrucción intestinal. En vista de que la diverticulistis de Meckel es indistinguible de otras condiciones inflamatorias intraabdominales más comunes es un diagnóstico diferencial que debemos considerar en caso de abdomen agudo.

\section{Cuadro No. 1: Diagnóstico Diferencial Diver- tículo de Meckel.}

\begin{tabular}{|l|l|l|}
\hline $\begin{array}{l}\text { Cuadro clínico } \\
\text { hemorrágico }\end{array}$ & $\begin{array}{l}\text { Cuadro clínico } \\
\text { obstructivo }\end{array}$ & $\begin{array}{l}\text { Cuadro clínico de } \\
\text { diverticulitis }\end{array}$ \\
\hline $\begin{array}{l}\text { Causas infecciosas: } \\
\text { Clostridium difficile, } \\
\text { Escherichia coli }\end{array}$ & $\begin{array}{l}\text { Intususcepción, } \\
\text { obstrucción } \\
\text { secundaria a } \\
\text { adherencias, } \\
\text { íleo, gastroente- } \\
\text { ritis o tumor. }\end{array}$ & $\begin{array}{l}\text { Apendicitis, } \\
\text { gastroenteritis, } \\
\text { adenitis } \\
\text { mesentérica }\end{array}$ \\
Angiodisplasias & $\begin{array}{l}\text { Patologías } \\
\text { ginecológicas: } \\
\text { torsión ovárica, } \\
\text { enfermedad } \\
\text { inflamatoria } \\
\text { pélvica }\end{array}$ \\
\hline $\begin{array}{l}\text { Hemorragia del trac- } \\
\text { to gastrointestinal }\end{array}$ & & \\
\hline
\end{tabular}

Fuente: Ruíz-Celorio M. Higuera-de la Tijera F. Pérez-Torres E. El divertículo de Meckel. Rev Med Hosp Gen Méx 2014;77(2): 88-92.

\section{BIBLIOGRAFÍA}

1. Arana J. Corona Bautista A. Vázquez Pelcastre R. Aparicio Ponce R. Divertículo de Meckel «invertido» como causa de oclusión intestinal: Reporte de un caso y revisión de la literatura. Evid Med Invest Salud 2014; 7 (1): 37-41

2. Ruíz-Celorio M. Higuera-de la Tijera F. Pérez-Torres E. El divertículo de Meckel. Rev Med Hosp Gen Méx 2014;77(2):88-92.

3. Martínez N. Pederzoli R. Sosa I. González E. Sanabria J. Frecuencia De Divertículo De Meckel En El Diagnostico Preoperatorio De La Apendicitis Aguda. Cir. Parag. 2015;
39 (2): 8-11.

4. Tas E. Culcu S. Duzkoylu Y. Eryilmaz S. Deniz M. Yilmaz D. A Rare Cause of Acute Abdomen: Perforation of Double Meckel's Diverticulum. Hindawi Publishing Corporation. Volume 2015, Article ID 648417, 4 pages. Revista en internet http://www. hindawi. com/journals/crigm/2015/648417/.

5. Mehta V. Speiser J. Eberhardt J. Yong S. Kapur U. Primary signet-ring cell carcinoma of Meckel's diverticulum: an uncommon cause of abdominal pain. Pathology, vol. 44, no. 4, pp. 365-367, 2012. 
6. Lee J, Kwag S. Oh S. Kim J. Kang W. Adenocarcinoma arising from Meckel's diverticulum in the ileum with malrotation of the midgut. Journal of the Korean Surgical Society, vol. 84, no. 6, pp. 367-370, 2013.

7. Thirunavukarasu P. Sathaiah M. Sukumar S. Bartels C. Zeh H. Lee K. et al. Meckel's Diverticulum-A High-Risk Region for Malignancy in the Ileum: Ann Surg. 2011; 253(2): 223-230.

8. Harmilten C. Arnason T. Ileitis associated with Meckel's diverticulum. Rev. Histopathology 2015;67:783-91.

9. Beyrouti M. Ben Amar M. Beyrouti R. Abid M. Ayadi L. Abid O. et al. Complications of Meckel's diverticulum. Report of 42 cases. Tunisie Medicale, vol. 87, no. 4, pp. 253-256, 2009.

10. Nael A. Wu M. Nagesh Rao P. Rezk S. Zhao X. Extranodal Marginal Zone Lymphoma Presenting within the Meckel Diverticulum as Diverticulitis: A Case Report. Hindawi. Volume 2014, Article ID 374814, 5 pages. Revista en internet http://www.hindawi.com/journals/cripa/2014/374814/

11. Sandoval García M. Rocha Millán J. Luisa Guadalupe Pineda Bahena L. Divertículo de Meckel, ¿un reto diagnóstico? Ferreiro Marín A. An Med (Mex) 2016; 61 (1): 68-72.

12. Prassad T. Churi C. Jacobsen A. Laparascopic resection of an axially torted Mecke'l Diverticulum in a 13-year-old Rev. Journal of Laparoendoscopi Advanced Sturgical Techniques 2005;16:425-427.

13. Clark J. koontz C. Smith L. Kelley J. Video-Assisted Transumbilical Meckel's diverticulectomy in children Rev. American Surgeon 2008;74: 327-329.
14. Fontenot B. Deutmeyer C. Hebra A. Volvular Small Bowet Obstruction Secondary to Adherence of a Meckel's Diverticulumat a previus Umbilical Laparascopic Por Site. Rev. Journal of Laparoendoscopi and Advance Surgical Techniques 2009;19: 251-254.

15. Sohn H. Park K. Lee, N. Lee S. Meckel's Diverticulum in exomphalos minor.

Rev. Annals of Surgical Treatment and Research 2015;91:90-92.

16. Yildhz I. Koca Y.S. Barut I. An unusual case of intraabdominal abscess and acute abdomen caused by axial torsion of meckel's diverticulum.

Rev. Annals of Medicine and Surgery 2016;6:74-76

17. Pepper V. Stangill A. Pearl R. Diagnosis and management of pediatric apendicitis, intussusception, and Meckel diverticulum. Surg Clin N Am 2012;92:505-526.

18. Labus P. Lazorčák M. Zofčák M. Meckel’s diverticulum in adults - our five-year experience. Rozhl Chir 2012;91:301-304.

19. Cotirlet A. Anghel R. Tincu E. Rau S. Motoc I. Popa E. Perforation of Meckel's diverticulum by foreign body, a rare complication. Chirurgia, vol. 108, pp. 411-413, 2013.

20. Liu X. Chan D.K. Tan K. Angioembolisation of a Bleeding Meckel's Diverticulum. Rev. Journal of Gastrointestinal Surgery 2015; 19:2286-7.

21. Bandi A. Tan Y. Tsang T. Correlation of gastric heterotopia and Meckel's diverticular bleeding in children: a unique association. Pediatr Surg Int 2014;30:313-316. 